\title{
Influenza vaccine effectiveness in preventing hospitalisation of individuals 60 years of age and over with laboratory-confirmed influenza, Valencia Region, Spain, influenza season 2016/17
}

Ainara Mira-Iglesias¹, F Xavier López-Labrador ${ }^{1,2}$, Beatriz Guglieri-López ${ }^{1}$, Miguel Tortajada-Girbés ${ }^{3}$, Víctor Baselga-Moreno ${ }^{1}$, Laura Cano ${ }^{1}$, Juan Mollar-Maseres ${ }^{4}$, Mario Carballido-Fernández ${ }^{5,6}$, Germán Schwarz-Chavarri7, Javier Díez-Domingo', Joan PuigBarberà $^{1,8}$, Valencia Hospital Network for the Study of Influenza and Respiratory Viruses Disease ${ }^{1}$

1. Fundación para el Fomento de la Investigación Sanitaria y Biomédica de la Comunitat Valenciana (FISABIO-Public Health), Valencia, Spain

2. Consorcio de Investigación Biomédica de Epidemiología y Salud Pública (CIBERESP), Instituto de Salud Carlos III, Madrid, Spain

3. Hospital Doctor Peset, Valencia, Spain

4. Hospital Universitario y Politécnico La Fe, Valencia, Spain

5. Hospital General Universitario de Castellón, Castellón, Spain

6. Universidad CEU Cardenal Herrera, Castellón, Spain

7. Hospital General de Alicante, Alicante, Spain

8. Centro de Salud Pública de Castellón, Castellón, Spain

Correspondence: Joan Puig-Barbera (jpuigb55@gmail.com)

Citation style for this article:

Mira-Iglesias Ainara, López-Labrador F Xavier, Guglieri-López Beatriz, Tortajada-Girbés Miguel, Baselga-Moreno Víctor, Cano Laura, Mollar-Maseres Juan,
Carballido-Fernández Mario, Schwarz-Chavarri Germán, Díez-Domingo Javier, Puig-Barberà Joan, Valencia Hospital Network for the Study of Influenza and

Respiratory Viruses Disease. Influenza vaccine effectiveness in preventing hospitalisation of individuals 60 years of age and over with laboratory-confirmed

influenza, Valencia Region, Spain, influenza season 2016/17. Euro Surveill. 2018;23(8):pii=17-00318. https://doi.org/10.2807/1560-7917.ES.2018.23.8.17-00318

Article submitted on 07 May 2017 / accepted on 01 Sep 2017 / published on 22 February 2018

Introduction: Seasonal influenza vaccination is widely recommended for people with risk factors, especially for people who are elderly. However, influenza vaccine effectiveness (IVE) varies year after year because of the variable antigenic composition of the circulating viruses and the vaccine composition. Methods: We summarise the results of IVE and the impact of previous vaccination among subjects 60 years of age and over in a multicentre prospective study in the Valencia Hospital Surveillance Network for the Study of Influenza and Respiratory Viruses Disease (VAHNSI) in Spain. We applied the test-negative design taking laboratory-confirmed influenza as outcome and vaccination status as exposure. Information about potential confounders was obtained from clinical registries or directly from patients. Results: Adjusted IVE was 19\% ( $95 \%$ confidence interval ( $\mathrm{Cl}):-15$ to 43 ). For patients vaccinated in the current season but not in the two previous seasons, effectiveness was $49 \%$ ( $95 \% \mathrm{Cl}:-20$ to 78) and for patients vaccinated in the current and any of two previous seasons, effectiveness was $29 \%$ (95\% $\mathrm{Cl}:-3$ to 52 ). For those patients not vaccinated in the current season but vaccinated in any of the two previous seasons, effectiveness was $53 \%$ (95\% Cl: 8 to 76). Conclusions: Our data show a low vaccine effectiveness for the 2016/17 influenza season.

\section{Introduction}

Vaccination is the primary recommendation for preventing seasonal influenza worldwide $[1,2]$. It is highly recommended in people who are elderly as they represent around $90 \%$ of all influenza-related deaths $[3,4]$. The World Health Organization (WHO) and the European Council encourage vaccination among risk groups to reach a coverage of $75 \%$ in people who are elderly and those with underlying chronic conditions [5].

The continuous evolution of influenza viruses means that health authorities have to reformulate the vaccine composition each year, trying to predict which strains will circulate in the coming influenza season. In order to contribute to improvement and better understanding of influenza vaccines, since 2009, an active annual surveillance scheme in the Valencia Region in Spain has been monitoring influenza vaccine effectiveness (IVE) in preventing hospital admissions with laboratory-confirmed influenza [6-8].

In the 2016/17 influenza season, a trivalent vaccine containing the recommended A/Hong Kong/4801/2014( $\left.\mathrm{H}_{3} \mathrm{~N}_{2}\right)$-like virus [9] was offered free of charge to all Valencia Region inhabitants 60 years of age over. However, the $2016 / 17$ season in the northern hemisphere was characterised by the circulation of $\mathrm{A}\left(\mathrm{H}_{3} \mathrm{~N}_{2}\right)$ viruses belonging to a new emerging 


\section{FIGURE 1}

Flowchart of hospital admissions 60 years of age and over included in the influenza vaccine effectiveness analysis, Valencia Hospital Network for the Study of Influenza (VAHNSI), Valencia Region, Spain, influenza season 2016/17 ( $=1,094)$

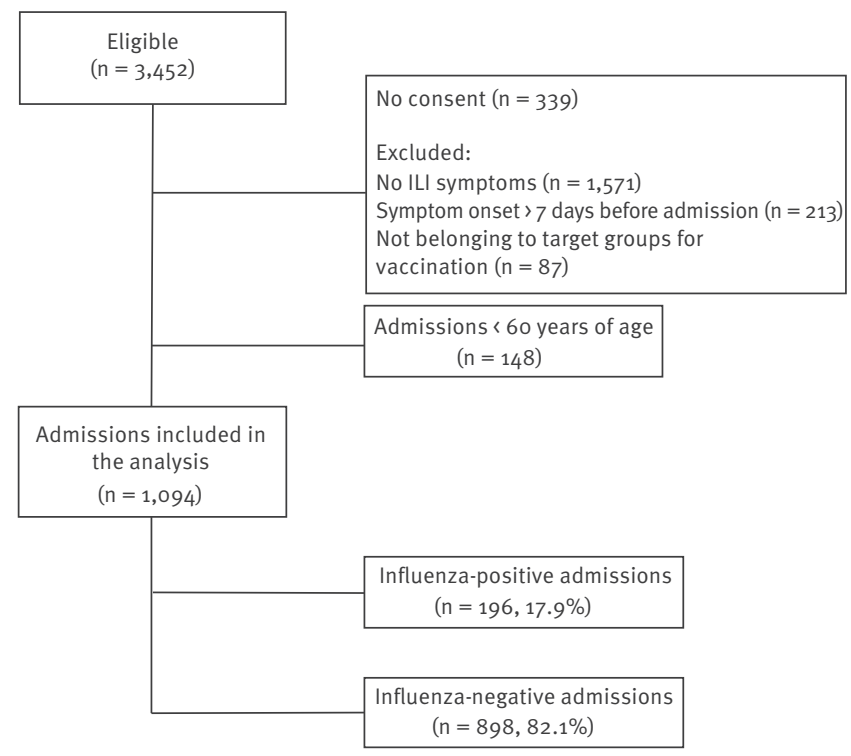

genetic subclade 3C.2a1, A/Bolzano/7/2016-like [10]. The new subclade is characterised by the $\mathrm{N} 171 \mathrm{~K}$ amino acid mutation as compared with the $\mathrm{A} /$ Hong Kong/4801/2014( $\left.\mathrm{H}_{3} \mathrm{~N}_{2}\right)$-like virus strain included in the northern hemisphere vaccine. In addition, significant amino acid heterogeneity in antigenic sites has been detected within this emerging subclade 3C.2a1 [11].

The interference between previous and current vaccination has been increasingly discussed. Castilla et al. [12] reported that whereas one or two vaccinations in the four prior seasons maintained or increased the protection of the current season vaccination, three or more doses had a negative effect on the current season vaccine. Other publications have suggested no protection with repeated vaccination, but that the current vaccination protected individuals unvaccinated in the previous season [13]. Similar results were obtained for the 2014/15 season in a school-based study in China [14] where children who had not received prior vaccination with a homologous $\mathrm{A}\left(\mathrm{H}_{3} \mathrm{~N}_{2}\right)$ component were more protected than children receiving repeated vaccination. Other studies have explored the impact of repeated vaccination on IVE against $A\left(\mathrm{H}_{3} \mathrm{~N}_{2}\right)$ influenza. Two of them concluded that current season's vaccination conferred either protection against medically attended influenza [15] or laboratory-confirmed hospitalised $A\left(\mathrm{H}_{3} \mathrm{~N}_{2}\right)$ influenza in people who are elderly [16], regardless of patients' recent vaccination history. In our study, we report IVE estimates in preventing hospital admissions with laboratory-confirmed influenza during the 2016/17 influenza season in the Valencia Region in Spain. The impact of prior vaccination is also evaluated considering the two previous influenza seasons.

\section{Methods}

\section{Study design}

The study was carried out in four hospitals providing healthcare to $22 \%$ of the $4,860,874$ inhabitants of Valencia Region in the east of Spain. The participating hospitals were: Hospital General de Castellón (Castellón, Spain), Hospital La Fe (Valencia, Spain), Hospital Doctor Peset (Valencia, Spain) and Hospital General de Alicante (Alicante, Spain).

Study procedures have been previously published [7]. In short, study staff screened consecutive hospital admissions through the emergency department. Patients were included in the study after written informed consent if they were a resident in the hospital catchment area, non-institutionalised, without a previous hospital discharge in the last 30 days and reported symptoms of influenza-like illness (ILI), defined as reported fever or feverishness, malaise, myalgia or headache and shortness of breath, sore throat or cough, within 7 days of admission. Subjects were considered immunised if they had received the current season's influenza vaccine at least 14 days before symptoms onset.

The Ethics Research Committee of the Dirección General de Salud Pública-Centro Superior de Investigación en Salud Pública (DGSP-CSISP) approved the protocol of the study. All patients signed informed consent before the inclusion in the study.

\section{Vaccine information system}

Information on the vaccine administered to all patients included in the study, in addition to the date of vaccination, was obtained from the Valencia Region Vaccine Information System (VRVIS). VRVIS is a populationbased register that systematically records vaccine doses given at public and private vaccination points, including primary care centres, hospitals, residential facilities in the public sector and any private sector facility that applies for access. The sensitivity and specificity of VRVIS was estimated to be $90 \%$ and $99 \%$, respectively $[7,17]$. All registered residents of Valencia Region have a unique identification number that is linked to the VRVIS, inpatient and outpatient clinical records, and sociodemographic information. Vaccine information was ascertained by recall in those patients whose vaccine administration was not registered in the system.

\section{Laboratory procedures}

Nasopharyngeal and pharyngeal swabs were collected from patients meeting the inclusion criteria and tested by real-time reverse transcription-PCR (RT-PCR) for influenza. The specimens were tested in a centralised virology laboratory at Fundación para el Fomento de la Investigación Sanitaria y Biomédica de la Comunitat Valenciana (FISABIO-Public Health) following World 
Distribution of influenza cases aged 60 years and over by influenza type/subtype and positivity rate by epidemiological week, Valencia Hospital Network for the Study of Influenza (VAHNSI), Valencia Region, Spain, influenza season $2016 / 17$ (n =196)

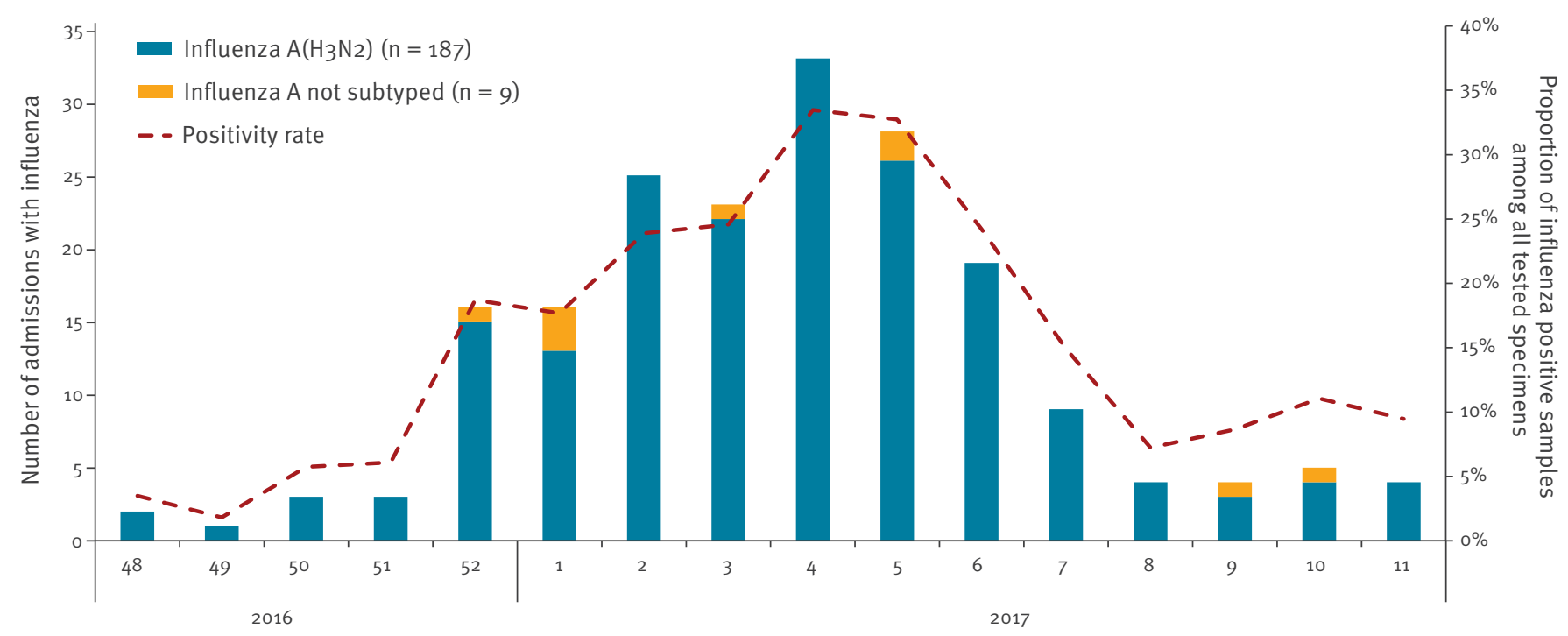

Week of admission

Health Organization (WHO) protocols [18]. In brief, one-third of the viral transport media volume in the combined swab sample tubes was extracted for total nucleic acids using an automated silica-based method (NucliSENS easyMAG, BioMérieux, Marcy-l'Etoile, France). A multiplex real-time RT-PCR screening assay was used to detect influenza $A$ and $B$ viruses using different primers and probes for the matrix protein $[19,20]$ and the qScript XLT One-Step RT-qPCR ToughMix (Quantabio, Beverly, Massachusetts, United States (US)) in a LightCycler 480ll apparatus (Roche Molecular Diagnostics, Sant Cugat, Spain).

For haemagglutinin ( $\mathrm{HA}$ ) sequencing, all isolates from hospitalised cases with sufficient viral load (cycle threshold $(\mathrm{Ct}) \leq 26)$ were systematically selected and a specific RT-PCR amplification protocol was applied using gene-specific primers for the corresponding virus subtype [21]. The amplified fragments were sequenced by the Sanger method with the BigDye Direct Cycle Sequencing Kit in an ABI 3730xl DNA Sequencer (Applied Biosystems, Life Technologies, Foster City, California, US) at the Genomics Core of the Servei Central de Suport a la Investigació Experimental (SCSIE), University of Valencia, Spain.

\section{Molecular phylogenetic analysis of influenza $A(H 3 N 2)$ viruses}

Because we detected no influenza $B$ viruses and only one $A\left(H_{1} N_{1}\right)$ pdmog virus during the $2016 / 17$ season, the phylogenetic characterisation was performed in $\mathrm{A}\left(\mathrm{H}_{3} \mathrm{~N}_{2}\right)$ viruses only. $\mathrm{A}$ dataset with $\mathrm{HA}$ sequences was constructed including the obtained influenza virus $\mathrm{HA}$ sequences together with representative and reference HA sequences (Supplement 1) obtained from the
Global Initiative on Sharing All Influenza Data (GISAID) database (www.gisaid.org). These representative sequences were chosen for specific amino acid signatures in the HA gene defining known phylogenetic clades. The dataset alignment was generated with the ClustalW algorithm integrated in the BioEdit software version 7.2.5 (http://www.mbio.ncsu.edu/bioedit/ bioedit.html). Phylogenetic trees were inferred using maximum likelihood methods and the best-fitting nt substitution model (general time-reversiblewith gamma distribution among sites) with the online PhyML 3.0 platform (http://www.atgc-montpellier.fr/ phyml). Branch reliability was evaluated by approximate likelihood-ratio tests [22]. The tree was rooted on the $\mathrm{A} /$ Perth/16/2009 $\left(\mathrm{H}_{3} \mathrm{~N}_{2}\right)$, clade $3 \mathrm{C}$ virus.

\section{Statistical analysis}

Characteristics of influenza-positive hospital admissions (cases) and influenza-negative hospital admissions (controls) were compared performing a chisquared test. We used the same test when comparing vaccinated and unvaccinated individuals. All probabilities were two-tailed and $p$ values under 0.05 were considered statistically significant.

The test-negative design was used to estimate the ratio of the odds of vaccination among individuals testing positive for influenza to the odds of vaccination among individuals testing negative. This approach has been described elsewhere [23,24] since it is less susceptible to bias due to misclassification of infection and to confounding by healthcare-seeking behaviour in comparison with case-control studies. Cases were patients testing positive for influenza and controls were patients who tested negative. The adjusted odds 


\section{FIGURE 3}

Phylogenetic tree of influenza A(H3N2) isolates, Valencia Hospital Network for the Study of Influenza (VAHNSI), Valencia Region, Spain, influenza season 2016/17

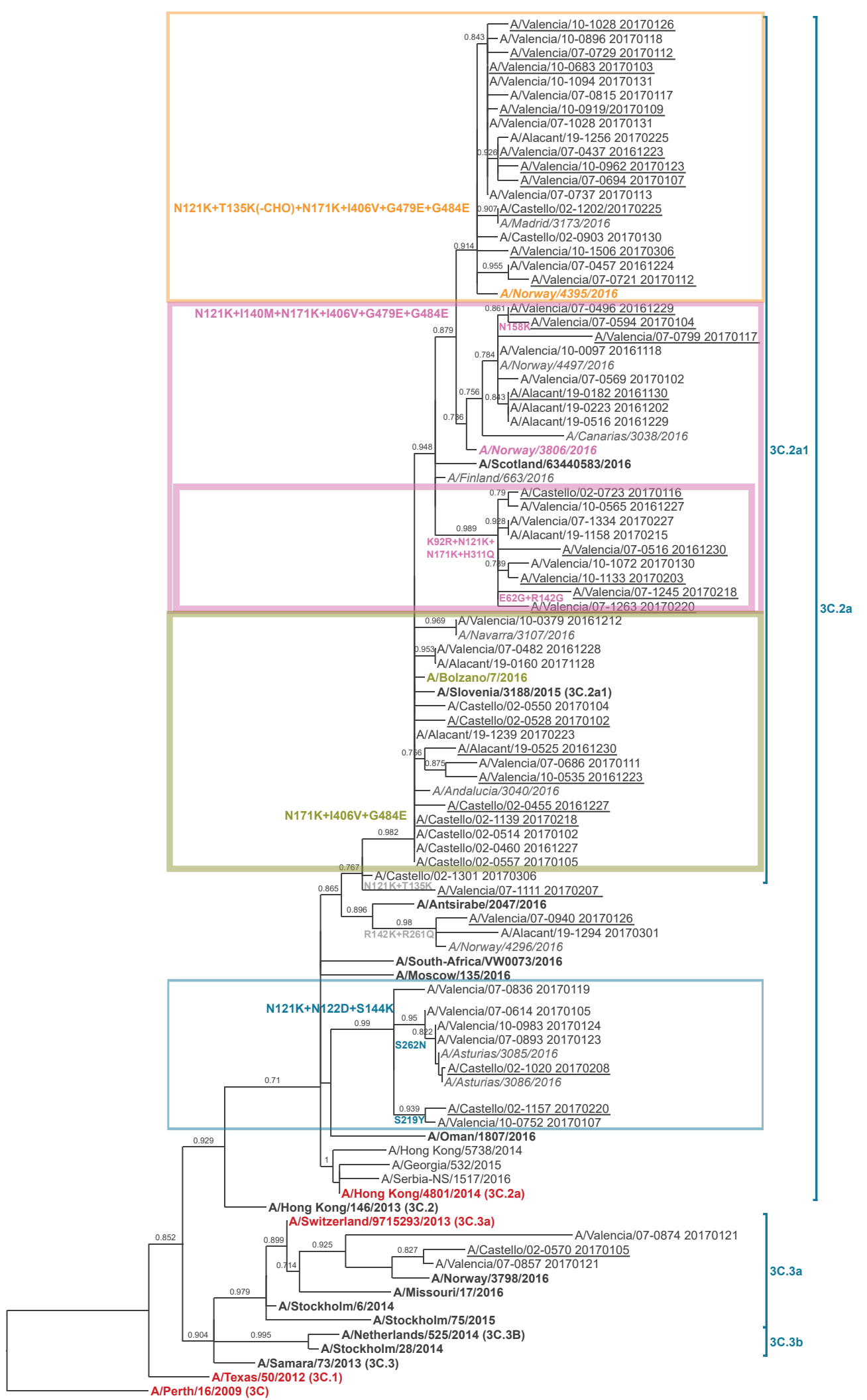

$\frac{1}{0.00 .001}$

Phylogenetic tree obtained by using maximum likelihood methods (general time-reversible substitution model with gamma distribution among sites) with PhyML 3.0. Branch reliability was evaluated by approximate likelihood-ratio tests and the tree was rooted on the A/Perth/16/2009( $\left.33 \mathrm{~N}_{2}\right)$, clade $3 \mathrm{C}$ virus. Reference sequences from the GISAID database. Red indicates vaccine strains, bold black indicates clade reference strains, italics represent European $\mathrm{A}\left(\mathrm{H}_{3} \mathrm{~N}_{2}\right)$ isolates circulating during the $2016 / 17$ influenza season, not related to this study. Local isolates from vaccinated individuals are underlined. The four amino acid variation patterns within subclade $3 C .2 a 1$ viruses are highlighted by different boxes. A new 3 C.2a local clade is highlighted by a blue coloured box.

The authors gratefully acknowledge the originating and submitting laboratories who contributed sequences used in the phylogenetic analysis to the Global Initiative on Sharing All Influenza Data (GISAID, www.gisaid.org). 
TABLE 1

Characteristics of hospital admissions aged 60 years and over included in the influenza vaccine effectiveness analysis, Valencia Hospital Network for the Study of Influenza (VAHNSI), Valencia Region, Spain, influenza season 2016/17 ( $\mathrm{n}=$ $1,094)$

\begin{tabular}{|c|c|c|c|c|c|c|c|c|c|}
\hline \multirow[t]{2}{*}{ Characteristics } & \multicolumn{2}{|c|}{$\begin{array}{l}\text { Influenza-positive } \\
\text { admissions (cases) }\end{array}$} & \multicolumn{2}{|c|}{$\begin{array}{l}\text { Influenza-negative } \\
\text { admissions (controls) }\end{array}$} & \multirow[t]{2}{*}{$p$ value ${ }^{a}$} & \multicolumn{4}{|c|}{ Vaccinated 2016/17 } \\
\hline & $n$ & $\%$ & $\mathrm{n}$ & $\%$ & & $n$ & Total & $\%$ & $\mathrm{p}$ value ${ }^{\mathrm{b}}$ \\
\hline Overall & 196 & 17.9 & 898 & 82.1 & - & 648 & 1,094 & 59.2 & - \\
\hline \multicolumn{10}{|l|}{ Age } \\
\hline $60-69$ years & 21 & 10.7 & 180 & 20.0 & \multirow{4}{*}{0.004} & 94 & 201 & 46.8 & \multirow{4}{*}{$<0.001$} \\
\hline 70-79 years & 62 & 31.6 & 304 & 33.9 & & 212 & 366 & 57.9 & \\
\hline $80-89$ years & 85 & 43.4 & 330 & 36.7 & & 267 & 415 & 64.3 & \\
\hline$\geq 90$ years & 28 & $14 \cdot 3$ & 84 & 9.4 & & 75 & 112 & 67.0 & \\
\hline \multicolumn{10}{|l|}{ Sex } \\
\hline Male & 93 & 47.4 & 475 & 52.9 & \multirow{2}{*}{0.167} & 341 & 568 & 60.0 & \multirow{2}{*}{0.574} \\
\hline Female & 103 & 52.6 & 423 & 47.1 & & 307 & 526 & 58.4 & \\
\hline \multicolumn{10}{|l|}{ Number of underlying chronic conditions } \\
\hline None & 26 & 13.3 & 110 & 12.2 & \multirow{3}{*}{0.628} & 62 & 136 & 45.6 & \multirow{3}{*}{$<0.001$} \\
\hline One & 59 & 30.1 & 246 & 27.4 & & 170 & 305 & 55.7 & \\
\hline Two or more & 111 & 56.6 & 542 & 60.4 & & 416 & 653 & 63.7 & \\
\hline \multicolumn{10}{|l|}{ Hospital admission in the last 12 months } \\
\hline Yes & 53 & 27.0 & 345 & 38.4 & \multirow{2}{*}{0.003} & 257 & 398 & 64.6 & \multirow{2}{*}{0.007} \\
\hline No & 143 & 73.0 & 553 & 61.6 & & 391 & 696 & 56.2 & \\
\hline \multicolumn{10}{|c|}{ Number of general practitioner consultations in the last 3 months } \\
\hline None & 28 & $14 \cdot 3$ & 103 & 11.5 & \multirow{3}{*}{0.090} & 65 & 131 & 49.6 & \multirow{3}{*}{$<0.001$} \\
\hline One & 49 & 25.0 & 177 & 19.7 & & 108 & 226 & 47.8 & \\
\hline Two or more & 119 & 60.7 & 618 & 68.8 & & 475 & 737 & 64.5 & \\
\hline \multicolumn{10}{|l|}{ Smoking habits } \\
\hline Never & 128 & 65.3 & 472 & 52.6 & \multirow{3}{*}{0.002} & 359 & 600 & 59.8 & \multirow{3}{*}{$<0.001$} \\
\hline Ex-smoker & 54 & 27.6 & 303 & 33.7 & & 228 & 357 & 63.9 & \\
\hline Current smoker & 14 & 7.1 & 123 & 13.7 & & 61 & 137 & 44.5 & \\
\hline \multicolumn{10}{|l|}{ Time from symptoms onset to swabbing } \\
\hline $0-2$ days & 26 & 13.3 & 144 & 16.0 & \multirow{4}{*}{0.108} & 109 & 170 & 64.1 & \\
\hline $3-4$ days & 97 & 49.5 & 359 & 40.0 & & 273 & 456 & 59.9 & 0.287 \\
\hline 5-7 days & 56 & 28.6 & 297 & 33.1 & & 205 & 353 & 58.1 & \\
\hline$>7$ days & 17 & 8.7 & 98 & 10.9 & & 61 & 115 & 53.0 & \\
\hline Influenza test results & & & & & & & & & \\
\hline Negative & - & - & 898 & 100.0 & & 537 & 898 & 59.8 & 0.414 \\
\hline $\mathrm{A}\left(\mathrm{H}_{3} \mathrm{~N}_{2}\right)$ & 187 & 95.41 & - & - & - & 107 & 187 & 57.22 & 0.538 \\
\hline A not subtyped & 9 & 4.59 & - & - & & 4 & 9 & 44.44 & 0.365 \\
\hline Vaccinated 2016/17 & 111 & 56.6 & 537 & 59.8 & 0.414 & - & - & - & - \\
\hline Vaccinated 2015/16 & 101 & 51.5 & 541 & 60.2 & 0.025 & 551 & 642 & 85.8 & $<0.001$ \\
\hline Vaccinated 2014/15 & 104 & 53.1 & 519 & 57.8 & 0.225 & 525 & 623 & 84.3 & $<0.001$ \\
\hline $\begin{array}{l}\text { Registration in the Valencia Region Vaccine } \\
\text { Information System }\end{array}$ & 161 & 82.14 & 786 & 87.5 & 0.045 & 643 & 947 & 67.9 & $<0.001$ \\
\hline
\end{tabular}

a $p$ value comparing influenza-positive admissions and influenza-negative admissions.

${ }^{\mathrm{b}} \mathrm{p}$ value comparing vaccinated with unvaccinated admissions.

ratio (aOR) was estimated using a mixed effects logistic regression model including age, sex, number of underlying chronic conditions, previous hospital admissions in the last 12 months, general practitioner (GP) consultations in the last 3 months, smoking habits, socioeconomic class according to occupation [25], days from onset of symptoms to swabbing and hospital as fixed effects, and epidemiological week at admission as random effect. Including epidemiological week as random effect allowed us to control for considerable extra variation that could be present across the different study weeks (number of working days, holidays and risk of infection). IVE was calculated as (1 - aOR) $\times$ 100. The same estimates were performed according to current and prior two seasons influenza vaccination 
taking as reference category no vaccination in any of the seasons.

All statistical analyses were carried out in Stata version 14 (StataCorp, College Station, Texas, US).

\section{Results}

\section{Study subjects}

The analysis was restricted to individuals 60 years of age and over because of small numbers in the other age groups. After applying exclusion criteria, 1,094 hospital admissions were included in the analysis (Figure 1). Vaccination was ascertained by recall (13\% of included patients) and by consulting the VRVIS ( $87 \%$ of included patients).

\section{Study period}

The beginning of the influenza season was defined as the first of two consecutive weeks with two or more influenza cases, and the end as the previous week to the first of two consecutive weeks with no influenza cases (Figure 2). This period was from week 48 of 2016 (starting 28 November) to week 11 (starting 13 March) of 2017. Our first influenza-positive patient was admitted on 29 November 2016 and our last influenza-positive patient was admitted 17 March 2017.

\section{Influenza cases vs controls}

A total of 196 hospital admissions (17.9\%) were positive for influenza A: 187 (95.4\%) were influenza $A\left(\mathrm{H}_{3} \mathrm{~N}_{2}\right)$ and $9(4.6 \%)$ remained unsubtyped because of low viral loads (Table 1 ).

Individuals testing positive for influenza were notably older than those testing negative. The percentage of admissions in hospital during the previous year among influenza-positive admissions was lower than the percentage among influenza-negative admissions. In terms of smoking habits, influenza-negative admissions were more likely to be current or ex-smokers than those who were influenza-positive. The proportion of vaccinated individuals in the $2015 / 16$ season was significantly higher in those testing negative for influenza (Table 1).

Vaccinated vs unvaccinated hospital admissions A total of $648(59.2 \%)$ hospital admissions were vaccinated for the 2016/17 influenza season. As expected, vaccination coverage increased significantly with age, but also with the number of underlying conditions. Vaccinated admissions were more likely admitted during the last year and to visit the GP in two or more occasions in the last 3 months. Admissions who never smoked or ex-smokers were more vaccinated than current smokers. Most of the admissions vaccinated in the current season were also vaccinated in the 2015/16 and in the 2014/15 influenza seasons (Table 1).
Molecular analysis

The genetic characterisation of the 63 sequenced influenza $A\left(\mathrm{H}_{3} \mathrm{~N}_{2}\right)$ samples indicated circulation of few clade 3C.3a, A/Switzerland/9715293/2013-like, viruses $(n=3)$, with the majority of isolates $(n=60)$, corresponding to the same clade $(3 \mathrm{C} .2 \mathrm{a})$ as the $\mathrm{A} /$ Hong Kong/4801/2014 vaccine strain for the $2016 / 17$ season (Figure 3).

However, $82 \%(n=49)$ of these clade 3 C.2a viruses belong to subclade $3 \mathrm{C} .2 \mathrm{a} 1$, all characterised by the $\mathrm{N} 171 \mathrm{~K}$ mutation as compared with the $\mathrm{A} / \mathrm{Hong}$ Kong/4801/2014 vaccine strain for the 2016/17 season (Figure 3). In addition, within this subclade 3C.2a1 we observed significant amino acid heterogeneity in antigenic sites, with up to four groups each characterised by a particular subset of mutations: (i) A/Bolzano/7/2006like group $(n=14)$ : $N 171 K\left(H_{1}\right), 1406 V+G 484 E\left(H A_{2}\right)$; (ii) a local cluster $(n=9)$ with additional mutations $\mathrm{K} 92 \mathrm{R}$ (site E) + N121K(site D) + $3311 \mathrm{Q}$ (site C) (HA1); (iii) A/Norway/3806/2016-like group $(n=8)$ : N121K(site D) $+1140 \mathrm{M}+\mathrm{N} 171 \mathrm{~K}(\mathrm{HA} 1), \quad \mathrm{l} 406 \mathrm{~V}+\mathrm{G} 479 \mathrm{E}+\mathrm{G} 484 \mathrm{E}\left(\mathrm{HA}_{2}\right)$; and (iv) $\quad A /$ Norway/4395/2016-like group $(n=18): \quad 121 K($ site $\quad D)+T_{135} K($ site $\quad A)+N 171 K(H A 1)$, $1406 V+G 479 E+G 484 E(H A 2)$; the latter with a potential loss of glycosylation at position 135 . Finally, the remaining eleven clade $3 \mathrm{C} .2 \mathrm{a}$ isolates were also genetically heterogeneous. Four were related to $\mathrm{A} /$ Antsirabe/2047/2016, with either mutations N121K(site D) $+\mathrm{T}_{135} \mathrm{~K}$ (site A) or R142K(site A) $+\mathrm{R} 261 \mathrm{Q}$ (site E), and the rest belonged to a new cluster characterised by the $\mathrm{N} 121 \mathrm{~K}($ site D) + N122D(site A) + $\mathrm{S} 144 \mathrm{~K}($ site $A)$ and either $\mathrm{S} 219 \mathrm{Y}$ or S262N(site E) (all HA1) mutations (Figure 3). The distribution of viral isolates from vaccinated individuals along the phylogenetic tree did not reveal any significant aggregation of cases with any particular virus genetic group.

\section{Influenza vaccine effectiveness and impact of prior vaccination}

Overall IVE in preventing hospital admissions with influenza was $18.7 \%$ ( $95 \%$ confidence interval (CI): -15.1 to 42.5 ) regardless of previous vaccination status. By age group, IVE was $37.0 \%$ (95\% Cl: -91.4 to $79.3)$ in $60-69$ years of age admissions, $-33.8 \%(95 \%$ $\mathrm{Cl}:-158.0$ to 30.6$)$ in those $70-79$ years of age, $27.3 \%$ ( $95 \% \mathrm{Cl}:-29.1$ to 59.1$)$ in those $80-89$ years of age and $61.0 \%$ ( $95 \% \mathrm{Cl}:-31.0$ to 88.4 ) in those 90 years of age and over (data not shown).

To explore the impact of prior vaccination, we took individuals unvaccinated in the $2016 / 17$ influenza season and two previous seasons as reference. For patients vaccinated in the current season but not in the two previous seasons, IVE was $48.6 \%$ ( $95 \% \mathrm{Cl}:-20.0$ to 78.0 ); for patients vaccinated in the current season and in either or in both of the two previous seasons, IVE was $29.4 \%$ ( $95 \% \mathrm{Cl}:-3.0$ to 51.6 ); and for those not vaccinated in the current season but in either or both of the two previous seasons, IVE was $53.5 \%$ (95\% Cl: 8.2 to 76.4) (Table 2). 
Adjusted influenza vaccine effectiveness against hospital admission of individuals 60 years old or of age and over with laboratory-confirmed influenza, Valencia Hospital Network for the Study of Influenza (VAHNSI), Valencia Region, Spain, influenza season 2016/17

\begin{tabular}{|c|c|c|c|c|c|c|}
\hline \multirow{2}{*}{ Analyses } & \multicolumn{2}{|c|}{ Cases $(n=196)$} & \multicolumn{2}{|c|}{ Controls $(n=898)$} & \multicolumn{2}{|c|}{ Adjusted IVE ${ }^{a}$} \\
\hline & Vaccinated & $\%$ & Vaccinated & $\%$ & VE \% & $95 \% \mathrm{Cl}$ \\
\hline Overall $2016 / 17^{b}$ & 111 & 56.6 & 537 & 59.8 & 18.7 & -15.1 to 42.5 \\
\hline NV in the previous two seasons, $V$ in the current season ${ }^{c}$ & 8 & 4.1 & 53 & 5.9 & 48.6 & -20.0 to 78.0 \\
\hline V in either or both previous two seasons, $V$ in the current season ${ }^{c}$ & 103 & 52.6 & 484 & 53.9 & 29.4 & -3.0 to 51.6 \\
\hline V in either or both previous two seasons, NV in the current season ${ }^{c}$ & 13 & 6.6 & 109 & 12.1 & 53.5 & 8.2 to 76.4 \\
\hline
\end{tabular}

V: vaccinated, NV: not vaccinated, IVE: influenza vaccine effectiveness.

a Adjusted by age, sex, number of underlying chronic conditions, previous hospital admissions in the last 12 months, general practitioner consultations in the last 3 months, smoking habits, socioeconomic class, days from onset of symptoms to swabbing and hospital as fixed effects, and epidemiological week at admission included as a random effect.

${ }^{b}$ Comparing vaccinated and unvaccinated admissions in the current influenza season.

Admissions unvaccinated in the current and previous two seasons taken as reference.

\section{Discussion}

The 2016/17 influenza season in the Valencia Region of Spain was dominated by influenza A viruses with almost all subtyped as $A\left(\mathrm{H}_{3} \mathrm{~N}_{2}\right)$. The positivity peak was earlier than those in recent years, and was higher than those observed in the 2015/16 and 2013/14 influenza seasons but similar to that observed in the 2014/15 season, which was also $\mathrm{A}\left(\mathrm{H}_{3} \mathrm{~N}_{2}\right)$-dominant [11]. Most of the genetically characterised viral isolates in Valencia Region belonged to a new emerging subclade, 3 C.2a1, evolving from the $\mathrm{A} / \mathrm{Hong} \mathrm{Kong} / 4801 / 2014\left(\mathrm{H}_{3} \mathrm{~N}_{2}\right)$ northern hemisphere 2016/17 influenza vaccine strain. Previous preliminary studies have also found these variant viruses in their analyses [26-28]. This emerging subclade has been reported as antigenically like the vaccine virus $[10,11,27]$ and preliminary IVE estimates have been moderate to low [12,26-28]. However, 3C.2a1 viruses are difficult to characterise antigenically as, due to accumulated mutations, there is a lack of appropriately matched reference antisera and agglutination capacity in haemagglutination assays is reduced [11].

Our results corroborated that this new emerging 3C.2a1 subclade was genetically heterogeneous. We distinguished at least three genetic subgroups with different amino acid mutation patterns in antigenic sites $A, C$ and $D$ as compared with the $A$ /Hong Kong/4801/2014 $\left(\mathrm{H}_{3} \mathrm{~N}_{2}\right)$ vaccine virus, one of them with the potential loss of a glycosylation site (T135K), which has also been found in Canada [26]. In addition, we identified a novel 3 C.2a local clade in Spain with three to four mutations in antigenic sites $A, D$ and $E$ (characterised by $\mathrm{N} 121 \mathrm{~K}+\mathrm{N} 122 \mathrm{D}+\mathrm{S} 144 \mathrm{~K}$ ) as compared with the vaccine strain, with potentially modified antigenicity. Interestingly, similar new 3 C.2a viruses have been characterised recently in the United Kingdom in the context of an outbreak [29].

Collectively, our results and available data published elsewhere suggest that clade 3 C.2a genetic diversification is ongoing, with rapid dynamics of different subclades. Although no significant changes in serological data have been detected yet, the diverse mutation patterns of new subclades suggest distinct mutant viruses can become predominant during the next 2017/18 season, whereas the recommended vaccine strain is still the same $A /$ Hong Kong/4801/2014 $\left(\mathrm{H}_{3} \mathrm{~N}_{2}\right)$-like virus. Whether the new predominant $\mathrm{A}\left(\mathrm{H}_{3} \mathrm{~N}_{2}\right)$ viruses in the next $2017 / 18$ season will be associated with changes in antigenicity and vaccine effectiveness deserves close monitoring, as the potential implications for Public Health may be important. The sensitivity to oseltamivir of the majority of the $2016 / 17 \mathrm{~A}\left(\mathrm{H}_{3} \mathrm{~N}_{2}\right)$ viruses may warrant antiviral management when necessary [11].

Our IVE analysis was restricted to admissions 60 years of age and over because of small numbers in the other age groups. Other studies reported that confirmed cases of influenza A were predominant in adults aged over 65 years of age with a substantial increase in mortality among individuals in this age group [26]. We observed a significant residual protection of previous vaccination and a lower effect in those vaccinated only in the current season or in the current and any of the two previous seasons. This pattern of protection was consistent with preliminary estimates in Europe [12,28].

Our results showed that IVE estimates were lower in the primary analysis, when no current vaccination rather than no current/no prior vaccination was used as reference group. This may be suggestive of residual protection (IVE for prior vaccination only). Interestingly, this residual protection may include vaccination with the previous $A\left(\mathrm{H}_{3} \mathrm{~N}_{2}\right)$ vaccine strain $A /$ Switzerland/9715293/2013 used in 2015/16 season, perhaps because of some cross-reactivity. Because of the residual protection observed, our findings do not support that a possible antigenic mismatch among the $\mathrm{A} /$ Hong Kong/4801/2014 vaccine strain and the 
2016/17 circulating strains influenced IVE according to the antigen distance hypothesis [30]. However, we and others [26-28] observed discrepancies between antigenic and vaccine effectiveness data that reinforce the consideration of genetic sequence analyses of influenza viruses [31].

Our study had several limitations. The results regarding the impact of repeated vaccination in the presence of genetic variation [32] were reassuring but should be interpreted with caution. The absence of statistical significance is expected in studies with low vaccine coverage, moderate to low effectiveness or a limited sample size resulting in wide confidence intervals [33]. The observational nature of our study meant that there was some heterogeneity in inpatient settings due to case ascertainment and exposure. However, we restricted the analysis to periods with influenza circulation and only included patients with ILI and an onset of symptoms within 7 days of admission to account for this heterogeneity. In addition, vaccination status was mostly ascertained with the information obtained from the VRVIS, and influenza positivity with a sensitive RT-PCR assay. Finally, we only included those patients with available swabbing performed within 48 hours of admission into hospital to avoid classification bias.

\section{Conclusion}

Although circulating influenza viruses were from the same genetic clade as the vaccine virus ( $A /$ Hong Kong/4801/2014), they showed a remarkable genetic heterogeneity with changes in antigenic sites. New 3C.2a subclades showed rapid and ongoing diversification, with important implications for the next 2017/18 influenza season because potential changes in antigenicity together with use of the same 2016/17 A/Hong Kong/4801/2014(H3N2)-like vaccine strain. Very early characterisation of $\mathrm{A}\left(\mathrm{H}_{3} \mathrm{~N}_{2}\right)$ viruses will be necessary for changes in vaccine effectiveness during the $2017 / 18$ influenza season [11], as shown by recent data from Canada [34].

The estimated IVE was low, although not significant, when comparing vaccinated and unvaccinated individuals in the current season regardless recent vaccination history. When considering prior vaccination, patients vaccinated in any of the two previous seasons but not in the current one were more protected than those individuals vaccinated in the current but not in the previous two seasons suggesting a residual protection obtained from previous vaccinations.

This work adds valuable information to the study of IVE and the controversial issue about the impact of repeated influenza vaccination. Our data may be especially relevant considering that vaccination against laboratory-confirmed influenza is being highly recommended in an increasingly number of countries worldwide.
Acknowledgements

We gratefully acknowledge the authors, originating and submitting laboratories who contributed the GISAID database sequences used in the phylogenetic analysis (Supplement 1) (www.gisaid.org).

The authors thank the staff of Hospital General de Castellón in Castellón; La Fe in Valencia; Hospital Dr Peset in Valencia; and Hospital General de Alicante in Alicante for their support. We thank all the study participants and their families.

Funding statement: The study was funded by a contract between FISABIO-Public Health and the Foundation for Influenza Epidemiology (FIE). The FIE did not participate in the design, conduct of the study, analysis or decision to publish the study. F Xavier López-Labrador also received funding from CIBERESP, Instituto de Salud Carlos III, Madrid, Spain.

\section{Conflict of interest}

None declared.

\section{Authors' contributions}

JPB: study coordinator, data analysis and writing manuscript. AMI and BGL: data analysis and writing manuscript. VBM: data management. FXLL and LC molecular analysis. All authors, including MTG, JMM, GSC, MCF and JDD, from the Valencia Hospital Network for the Study of Influenza (VAHNSI), contributed to the conception and design of the work; the acquisition, analysis, and interpretation of data; revised the manuscript and approved the final version.

\section{References}

1. World Health Organization (WHO). Vaccines against influenza WHO position paper - November 2012. Wkly Epidemiol Rec. 2012;87(47):461-76. PMID: 23210147

2. Gasparini R, Lucioni C, Lai P, Maggioni P, Sticchi L, Durando P, et al. Cost-benefit evaluation of influenza vaccination in the elderly in the Italian region of Liguria. Vaccine. 2002;20(Suppl 5):B50-4. https://doi.org/10.1016/S0264-410X(02)00507-8 PMID: 12477420

3. Simonsen L, Clarke MJ, Schonberger LB, Arden NH, Cox NJ, Fukuda K. Pandemic versus epidemic influenza mortality: a pattern of changing age distribution. J Infect Dis. 1998;178(1):53-60. https://doi.org/10.1086/515616 PMID: 9652423

4. Simonsen L, Reichert TA, Viboud C, Blackwelder WC, Taylor RJ, Miller MA. Impact of influenza vaccination on seasonal mortality in the US elderly population. Arch Intern Med. 2005;165(3):265-72. https://doi.org/10.1001/ archinte.165.3.265 PMID: 15710788

5. Council of the European Union. Council recommendation of 22 December 2009 on seasonal influenza vaccination. Official Journal of the European Union. Luxembourg: Publications Office of the European Union; 2009. Available from: http://eurlex.europa.eu/legal-content/EN/TXT/HTML/?uri=CELEX:32009 $\mathrm{H} 1019$ \& from $=\mathrm{EN}$

6. Puig-Barberà J, Arnedo-Pena A, Pardo-Serrano F, TiradoBalaguer MD, Pérez-Vilar S, Silvestre-Silvestre E, et al. Effectiveness of seasonal 2008-2009, 2009-2010 and pandemic vaccines, to prevent influenza hospitalizations during the autumn 2009 influenza pandemic wave in Castellón, Spain. A test-negative, hospital-based, case-control study. Vaccine. 2010;28(47):7460-7. https://doi.org/10.1016/j. vaccine.2010.09.042 PMID: 20875486

7. Puig-Barberà J, García-de-Lomas J, Díez-Domingo J, ArnedoPena A, Ruiz-García M, Limón-Ramírez R, et al. Influenza vaccine effectiveness in preventing influenza $A\left(\mathrm{H}_{3} \mathrm{~N}_{2}\right)$-related hospitalizations in adults targeted for vaccination by type of vaccine: a hospital-based test-negative study, 2011-2012 $\mathrm{A}\left(\mathrm{H}_{3} \mathrm{~N}_{2}\right)$ predominant influenza season, Valencia, Spain. PLoS One. 2014;9(11):e112294. https://doi.org/10.1371/journal. pone.0112294 PMID: 25392931 
8. Puig-Barberà J, Mira-Iglesias A, Tortajada-Girbés M, LopezLabrador FX, Belenguer-Varea A, Carballido-Fernandez M, et al. Effectiveness of influenza vaccination programme in preventing hospital admissions, Valencia, 2014/15 early results. Euro Surveill. 2015;20(8):21044. https://doi. org/10.2807/1560-7917.ES2015.20.8.21044 PMID: 25742432

9. World Health Organization (WHO). Recommended composition of influenza virus vaccines for use in the 2016-2017 northern hemisphere influenza season. Wkly Epidemiol Rec. 2016;91(10):121-32. PMID: 26971356

10. European Centre for Disease Prevention and Control (ECDC). Risk assessment of seasonal influenza, EU/EEA, 2016/2017 24 December 2016. Stockholm: ECDC; 26 Dec 2016. Available from: https://ecdc.europa.eu/en/publications-data/ risk-assessment-seasonal-influenza-eueea-20162017-24december-2016

11. The Francis Crick Institute. Reported prepared for the WHO annual consultation on the composition of influenza vaccine for the Northern Hemisphere 2017-2018. London: The Francis Crick Institute;2017. Available from: https://www.crick.ac.uk/ media/358671/crick_nh_vcm_report_feb_2017_v2.pdf

12. Castilla J, Navascués A, Casado I, Díaz-González J, PérezGarcía A, Fernandino L, et al. Combined effectiveness of prior and current season influenza vaccination in northern Spain: 2016/17 mid-season analysis. Euro Surveill. 2017;22(7):30465. https://doi.org/10.2807/1560-7917.ES.2017.22.7.30465 PMID: 28230523

13. Ohmit SE, Petrie JG, Malosh RE, Cowling BJ, Thompson MG, Shay DK, et al. Influenza vaccine effectiveness in the community and the household. Clin Infect Dis. 2013;56(10):1363-9. https://doi.org/10.1093/cid/cito6o PMID: 23413420

14. Zhang L, Yang P, Thompson MG, Pan Y, Ma C, Wu S, et al. Influenza vaccine effectiveness in preventing influenza illness among children during school-based outbreaks in the 2014-2015 season in Beijing, China. Pediatr Infect Dis J. 2017;36(3):e69-75. https://doi.org/10.1097/ INF.0000000000001434 PMID: 27902651

15. McLean HQ, Thompson MG, Sundaram ME, Meece JK, McClure $\mathrm{DL}$, Friedrich TC, et al. Impact of repeated vaccination on vaccine effectiveness against influenza $A\left(\mathrm{H}_{3} \mathrm{~N}_{2}\right)$ and $B$ during 8 seasons. Clin Infect Dis. 2014;59(10):1375-85. https://doi. org/10.1093/cid/ciu680 PMID: 25270645

16. Rondy M, Launay 0, Castilla J, Costanzo S, Puig-Barberà J, Gefenaite G, et al. Repeated seasonal influenza vaccination among elderly in Europe: Effects on laboratory confirmed hospitalised influenza. Vaccine. 2017;35(34):4298-306. https://doi.org/10.1016/j.vaccine.2017.06.088 PMID: 28709555

17. Puig-Barberà J, Natividad-Sancho A, Calabuig-Pérez J, LluchRodrigo JA, Pastor-Villalba E, Martínez-Úbeda S, et al. MF59adjuvanted and virosomal influenza vaccines for preventing influenza hospitalization in older people: comparative effectiveness using the Valencia health care information system. Vaccine. 2013;31(37):3995-4002. https://doi. org/10.1016/j.vaccine.2013.05.070 PMID: 23731629

18. World Health Organization (WHO). WHO information for molecular diagnosis of influenza virus in humans - update. Geneva: WHO; Nov 2012. [Accessed 12 Feb 2018]. Available from: http://www.who.int/influenza/gisrs_laboratory/ molecular diagnosis influenza virus humans update_201211.pdf

19. He J, Bose ME, Beck ET, Fan J, Tiwari S, Metallo J et al. Rapid multiplex reverse transcription-PCR typing of influenza $A$ and $B$ virus, and subtyping of influenza $A$ virus into $\mathrm{H}_{1}, 2,3,5,7,9$, $\mathrm{N}_{1}$ (human), N1 (animal), N2, and N7, including typing of novel swine origin influenza $A\left(\mathrm{H}_{1} \mathrm{~N}_{1}\right)$ virus, during the 2009 outbreak in Milwaukee, Wisconsin. J Clin Microbiol. 2009;47(9):2772-8. https://doi.org/10.1128/JCM.00998-09 PMID: 19641063

20. Suwannakarn K, Payungporn S, Chieochansin T, Samransamruajkit R, Amonsin A, Songserm T, et al. Typing $(A / B)$ and subtyping $\left(\mathrm{H}_{1} / \mathrm{H}_{3} / \mathrm{H}_{5}\right)$ of influenza $A$ viruses by multiplex real-time RT-PCR assays. J Virol Methods. 2008;152(12):25-31. https://doi.org/10.1016/j.jviromet.2008.06.002 PMID: 18598722

21. World Health Organization (WHO). WHO molecular diagnosis of influenza virus in humans - update. Geneva: WHO; Aug 2011. [Accessed Feb 2018]. Available from: http://www.who. int/influenza/resources/documents/molecular_diagnosis_ influenza_virus_humans_update_201108.pdf

22. Guindon S, Dufayard JF, Lefort V, Anisimova M, Hordijk W, Gascuel $\mathrm{O}$. New algorithms and methods to estimate maximum-likelihood phylogenies: assessing the performance of PhyML 3.0. Syst Biol. 2010;59(3):307-21. https://doi. org/10.1093/sysbio/syq010 PMID: 20525638

23. Jackson ML, Nelson JC. The test-negative design for estimating influenza vaccine effectiveness. Vaccine. 2013;31(17):2165-8. https://doi.org/10.1016/j.vaccine.2013.02.053 PMID: 23499601

24. Sullivan SG, Feng S, Cowling BJ. Potential of the test-negative design for measuring influenza vaccine effectiveness: a systematic review. Expert Rev Vaccines. 2014;13(12):157191. https://doi.org/10.1586/14760584.2014.966695 PMID: 25348015

25. Galobardes B, Shaw M, Lawlor DA, Lynch JW, Davey Smith G. Indicators of socioeconomic position (part 2). J Epidemiol Community Health. 2006;60(2):95-101. https://doi. org/10.1136/jech.2004.028092 PMID: 16415256

26. Skowronski DM, Chambers C, Sabaiduc S, Dickinson JA, Winter AL, De Serres G, et al. Interim estimates of 2016/17 vaccine effectiveness against influenza $\mathrm{A}\left(\mathrm{H}_{3} \mathrm{~N}_{2}\right)$, Canada, January 2017. Euro Surveill. 2017;22(6):30460. https://doi. org/10.2807/1560-7917.ES.2017.22.6.30460 PMID: 28205503

27. Flannery B, Chung JR, Thaker SN, Monto AS, Martin ET, Belongia EA, et al. Interim Estimates of 2016-17 Seasonal Influenza Vaccine Effectiveness - United States, February 2017. MMWR Morb Mortal Wkly Rep. 2017;66(6):167-71. https://doi. org/10.15585/mmwr.mm6606a3 PMID: 28207689

28. Kissling E, Rondy MI-MOVE/I-MOVE+ study team. Early 2016/17 vaccine effectiveness estimates against influenza $A\left(\mathrm{H}_{3} \mathrm{~N}_{2}\right)$ : I-MOVE multicentre case control studies at primary care and hospital levels in Europe. Euro Surveill. 2017;22(7):30464. https://doi.org/10.2807/1560-7917.ES.2017.22.7.30464 PMID: 28230524

29. Harvala H, Frampton D, Grant P, Raffle J, Ferns RB, Kozlakidis $Z$, et al. Emergence of a novel subclade of influenza $A\left(\mathrm{H}_{3} \mathrm{~N}_{2}\right)$ virus in London, December 2016 to January 2017. Euro Surveill. 2017;22(8):30466. https://doi.org/10.2807/1560-7917. ES.2017.22.8.30466 PMID: 28251889

30. Skowronski DM, Chambers C, De Serres G, Sabaiduc S, Winter $A L$, Dickinson JA, et al. Serial vaccination and the antigenic distance hypothesis: effects on influenza vaccine effectiveness during $\mathrm{A}\left(\mathrm{H}_{3} \mathrm{~N}_{2}\right)$ epidemics in Canada, 2010-11 to 2014-15. J Infect Dis. 2017;215(7):1059-99. https://doi.org/10.1093/ infdis/jix074 PMID: 28180277

31. Katz JM, Hancock K, Xu X. Serologic assays for influenza surveillance, diagnosis and vaccine evaluation. Expert Rev Anti Infect Ther. 2011;9(6):669-83. https://doi.org/10.1586/eri.11.51 PMID: 21692672

32. Skowronski DM, Chambers C, Sabaiduc S, De Serres G, Winter $A L$, Dickinson JA, et al. A perfect storm: Impact of genomic variation and serial vaccination on low influenza vaccine effectiveness during the 2014-2015 season. Clin Infect Dis. 2016;63(1):21-32. https://doi.org/10.1093/cid/ciw176 PMID: 27025838

33. Ellis PD. The Essential Guide to Effect Sizes: Statistical Power, Meta-Analysis, and the Interpretation of Research Results. Cambridge: Cambridge University Press; 2010.

34. Skowronski DM, Chambers C, De Serres G, Dickinson JA, Winter A-L, Hickman R, et al. Early season co-circulation of influenza $\mathrm{A}\left(\mathrm{H}_{3} \mathrm{~N}_{2}\right)$ and $\mathrm{B}$ (Yamagata): interim estimates of $2017 / 18$ vaccine effectiveness, Canada, January 2018. Euro Surveill. 2018;23(5):18-00035. https://doi.org/10.2807/15607917.ES.2018.23.5.18-00035 PMID: 29409570

\section{License and copyright}

This is an open-access article distributed under the terms of the Creative Commons Attribution (CC BY 4.0) Licence. You may share and adapt the material, but must give appropriate credit to the source, provide a link to the licence, and indicate if changes were made.

This article is copyright of the authors, 2018. 